\title{
Fungal colonization in patients with chronic respiratory diseases from Himalayan region of India
}

Debasis Biswas ${ }^{1 *}$, Sonal Agarwall, Girish Sindhwani ${ }^{2}$, Jagdish Rawat ${ }^{2}$

\begin{abstract}
Background: We screened patients with chronic respiratory diseases for microbiological and serological evidences of fungal colonisation; in order to determine its prevalence in this group of patients, examine potential clinical and radiological predictors of fungal colonisation and characterise fungal agents associated with individual diseases.

Methods: BAL samples from 60 consecutive patients were subjected to microscopy and culture for fungal agents, Serum samples were analysed for precipitin antibodies to Aspergillus antigen and Candida cytoplasmic antigen. Statistical significance in the difference of fungal recovery between patient groups was determined using the Chisquare test.

Results: The major diagnostic groups included patients with bronchogenic carcinoma $(n=31)$ and tubercular sequelae $(n=16)$. In all, 28 patients $(46.7 \%)$ were culture-positive, with Candida and Aspergillus being recovered from 14 and 13 patients respectively. Twenty-one patients (35\%) showed presence of precipitin antibodies. Patients with bronchogenic carcinoma showed increased predilection for colonisation with Aspergillus, while Candida was recovered more commonly in tubercular sequelae $(p=0.02)$. There was no statistically significant association between culture-positivity and specific risk factors/radiological findings.

Conclusion: The point-prevalence of fungal colonization was almost 50\%. The combination of fungal culture and serology helped improve diagnostic sensitivity. An interesting predilection was observed for Aspergillus and Candida, to preferentially infect patients with Bronchogenic carcinoma and Tubercular sequelae respectively. In absence of specific predictors, the possibility of fungal colonization needs to be explored actively in these patients.
\end{abstract}

\section{Background}

Fungal infections have emerged as a world-wide health care problem in recent years [1], owing to the extensive use of broad-spectrum antibiotics [2], long-term use of immunosuppressive agents, increasing use of hyperalimentation and indwelling devices [3] and the increasing population of terminally ill, debilitated and immunocompromised patients [4]. In tune with this general trend, there has been a phenomenal rise in the occurrence of fungal lung infections over the last two decades [5], a significant fraction of which is communityacquired [1]. Specific diagnosis of fungal pneumonia assumes importance in view of the different therapeutic

\footnotetext{
*Correspondence: dbiswas71@rediffmail.com

'Deptartment of Microbiology, Himalayan Institute of Medical Sciences,

Swami Ram Nagar, Jolly Grant, Dehradun, Uttarakhand, 248140, India

Full list of author information is available at the end of the article
}

strategies involved and the higher mortality associated with acute invasive fungal infection. Unfortunately, diagnosis of fungal respiratory infection is always difficult, owing to the lack of pathognomonic clinical features, contamination of the non-invasive samples like sputum with normal commensal flora and difficulty in obtaining invasive samples like translaryngeal aspirate and lung biopsy. A previous study has shown that Candida colonization could be found in the respiratory samples obtained by Bronchoalveolar lavage (BAL), endotracheal aspirate or protected specimen brushing in critically ill patients [6]. Since patients with chronic lung pathology provide a suitable nidus for fungal colonization, we hypothesized that screening such patients for fungal colonization of the respiratory tract would enable us to identify individuals requiring closer monitoring for the development of possible complications like acute 
invasive fungal infection or dissemination via hematogenous spread. The present study was designed with the purpose of characterizing the fungal pathogens associated with particular pulmonary diseases, and correlating between the individual fungal pathogens and the profile of risk factors, radiological presentations and clinical conditions in these patients.

\section{Methods}

Recruitment of patients and collection of clinical samples The study extended over a period of 12 months and included 60 consecutive patients with chronic lung diseases who required bronchoscopic examination for diagnostic purposes. Patients suffering from active Tuberculosis, pneumonitis and HIV-positive patients were excluded. The study protocol was duly approved by the institutional research and ethics committees. Signed informed consent was obtained from all the study participants.

Bronchoalveolar lavage (BAL) sample was collected aseptically with the help of flexible bronchoscope (Olympus Corporation; BF type TE2), attached to a light source (Olympus CLK-4) and digital signal processing camera. The selection of the site for collection of BAL fluid was guided by prior imaging studies and local inspection of the disease site. As advocated by Jourdain et al no endobronchial suction was attempted during the advancement of the bronchoscope to avoid contamination with upper airway flora and the first two aliquots were discarded [7]. The BAL fluid was collected in a sterile container and transported immediately to the mycology laboratory. None of the patients had received antibiotics for at least 2 weeks prior to the collection of BAL fluid. Serum samples were also collected from the same patients and stored at $-20^{\circ} \mathrm{C}$ for serological analysis.

\section{Mycological processing}

Centrifuged deposits prepared from BAL samples were used for direct microscopy and culture on Sabouraud's dextrose agar (SDA) with and without antibiotics and Czapek Dox Agar. The clinical significance of these isolates were determined by ensuring that contamination with oral flora had not taken place during sampling and by correlating the culture record with observation made during direct microscopy. Moreover, the growth was considered to be significant only when the same fungal agent was isolated from the different culture tubes.

The significant fungal isolates recovered on culture were identified to the species level, using standard mycological procedures. For Candida $s p$. isolates, the tests performed included germ tube test, Dalmau culture plate, sugar assimilation and fermentation tests and ChromAgar. Identification of Aspergillus sp. was done by examining the colony morphology and microscopic morphology of wet mounts prepared from the fungal growth, before and after slide culture examination. Cryptococcus neoformans was identified by characteristic colony morphology, India ink preparation

\section{Immunodiffusion test with Candida and Aspergillus antigens}

For immunodiffusion tests, Aspergillus antigen and antisera were commercially procured (Adaltis Inc, Italy) and Candida cytoplasmic antigen and corresponding antisera were kindly provided by Prof. Arunaloke Chakrabarti, Deptt of Medical Microbiology, Post Graduate Institute of Medical Education \& Research, Chandigarh, India. Double diffusion test was done at $25^{\circ} \mathrm{C}$ by Oudin-Ouchterlony's agar gel diffusion method and the immunodiffusion plates were observed for a week to detect the presence of precipitin bands. On appearance, the precipitin bands were stained in amido black solution.

\section{Statistical analysis}

The Chi-square test was used to examine if there was statistically significant difference in the fungal recovery rates between patients belonging to different diagnostic categories and having different co-morbid conditions or risk factors. Chi-square test was also used to analyse possible association between fungal recovery and specific radiological findings in Chest X-ray. Yates' corrected Chi-square test was employed to analyse differences in the nature of fungal isolates recovered from patients with Bronchogenic carcinoma and tubercular sequelae.

\section{Results}

\section{Clinical profile of patients}

The study group comprised of a total of 60 patients (44 males) with ages ranging from 40 to 85 years (median age being 55 years). Thirty one patients $(51.7 \%)$ presented with bronchogenic carcinoma, while 16 patients (26.7\%) had tubercular sequelae like persisting cavity, collapse, fibrosis, etc. Among the different co-morbid conditions and risk factors, the common associations included diabetes mellitus (DM), severe anaemia ( $\mathrm{Hb}$ $<9 \%$ ), use of steroids, alcoholism and chronic carrier state of HBV. While 10 patients had normal chest $\mathrm{X}$-ray, radiological abnormalities were detected in 50 patients, with consolidation $(n=10)$, collapse $(n=9)$ and nodular lesion $(\mathrm{n}=8)$ being the common findings.

\section{Recovery of fungal agents}

Direct microscopic examination revealed the presence of septate hyphae with dichotomous branching in 10 patients and budding yeast cells in 11 patients. Fungal culture yielded Aspergillus sp. in 13 patients (including A. flavus in 6 patients, A. fumigatus in 4 patients and $A$. 
niger in 3 patients) and Candida sp. in 14 (C. albicans and $C$. tropicalis being isolated from 12 and 2 patients respectively). One patient, with chronic interstitial lung disease, showed growth of Cryptococcus neoformans. The distribution of the fungal isolates with respect to the diagnostic groups is represented in Table 1. In all, 28 patients (46.7\%) were culture-positive.

\section{Detection of anti-fungal precipitin antibodies}

Twenty one of the recruited patients (35\%) reacted positively in the immunodiffusion assays. Of the 13 and 14 patients who showed growth of Aspergillus sp. and Candida sp. in their BAL samples, 7 (53.8\%) and 8 (57.1\%) patients respectively were positive for the corresponding anti-fungal antibodies in the immunodiffusion assay. Similarly among the serum samples from 47 and 46 culture-negative patients, $45(95.7 \%)$ and $42(91.3 \%)$ patients respectively were found to be negative for precipitin antibodies. Hence, taking culture as the gold standard, immunodiffusion had a sensitivity of $53.8 \%$ and a specificity of $95.7 \%$. The positive and negative predictive values of immunodiffusion assay were $77.7 \%$ and $88.2 \%$ respectively. The corresponding figures for detection of anti-Candida antibodies were $57.1 \%, 91.3 \%, 66.7 \%$ and $87.5 \%$ respectively.

\section{Correlation of microbiological and serological evidences of fungal infection}

In all, 15 of the 27 culture-positive patients (55.5\%) were also positive in immunodiffusion tests and culture-positivity, in absence of precipitin antibodies, was observed in 12 patients. Eight of these 12 patients had co-morbid immunosuppressive factors, like DM (3 patients), cancer chemotherapy ( 2 patients), steroid use (1 patient), chronic renal failure (1 patient) and chronic carriage of HBV (I patient). Six patients were positive for precipitin bands, despite being culture-negative. Interestingly, 5 of these 6 patients had bronchogenic carcinoma, while the sixth patient had pleural effusion of unresolved aetiology (Table 2).

\section{Clinical and radiological associates of fungal infection}

We next analysed whether the incidence of fungal colonization in the recruited patients had any association with their clinical condition, presence of specific risk factors or radiological findings. Though the difference in culture-positivity rates between patients with Bronchogenic carcinoma and tubercular sequelae was statistically non-significant $(\mathrm{p}=0.4)$, the nature of fungal isolates were different between the two clinical conditions. Among patients with Bronchogenic carcinoma, 8 patients showed growth of Aspergillus sp. while Candida $s p$. was recovered from 2 . In contrast, among patients with tubercular sequelae, Candida $s p$. was recovered from 6 patients while only a single patient showed growth of Aspergillus sp. This difference in the nature of fungal pathogens recovered from patients with Bronchogenic carcinoma and tubercular sequelae was statistically significant ( $\mathrm{p}=0.02$; Yates' corrected Chi-square test).

Diabetes mellitus was found to be the commonest risk factor associated with the isolation of Candida albicans. Out of 12 patients having Candida albicans as a fungal isolate, 5 were having diabetes mellitus as a predisposing factor (Table 3). However, there was no statistically

Table 1 Recovery of fungal agents with respect to clinical diagnosis

\begin{tabular}{|c|c|c|c|c|}
\hline Clinical Diagnosis & No. of patients & Culture +ve patients (\%) & Isolates & No. of Isolates (\%) \\
\hline \multirow[t]{4}{*}{ Bronchogenic carcinoma } & 31 & $10(32.3)$ & A. flavus & $5(16.1)$ \\
\hline & & & C. albicans & $2(6.4)$ \\
\hline & & & A. niger & $2(6.4)$ \\
\hline & & & A. fumigatus & $1(3.2)$ \\
\hline \multirow[t]{3}{*}{ Tubercular sequelae } & 16 & $7(43.8)$ & C. albicans & $5(31.3)$ \\
\hline & & & C. tropicalis & $1(6.3)$ \\
\hline & & & A. fumigatus & $1(6.3)$ \\
\hline \multirow[t]{3}{*}{ Bronchiectasis } & 5 & $5(100)$ & C. albicans & $3(60)$ \\
\hline & & & A. flavus & $1(20)$ \\
\hline & & & A. fumigatus & $1(20)$ \\
\hline Pleural effusion & 2 & $1(50)$ & A. niger & $1(50)$ \\
\hline \multirow[t]{2}{*}{ Bronchial asthma } & 2 & $2(100)$ & C. albicans & $1(50)$ \\
\hline & & & A. fumigatus & $1(50)$ \\
\hline \multirow[t]{2}{*}{ COPD } & 3 & $2(66.7)$ & C. albicans & $1(33.3)$ \\
\hline & & & C. tropicalis & $1(33.3)$ \\
\hline Interstitial lung disease & 1 & $1(100)$ & C. neoformans & $1(100)$ \\
\hline
\end{tabular}


Table 2 Results of Immunodiffusion assay for fungus-specific antibodies

\begin{tabular}{ccccc}
\hline & Results of Immnodiffusion assay & Culture positive & \multicolumn{2}{c}{ Culture negative } \\
\hline Aspergillus-specific antibodies & Immunodiffusion positive & 7 & 2 & 9 \\
\cline { 2 - 5 } & Immunodiffusion negative & 6 & 45 & 51 \\
\cline { 2 - 5 } & Total & 13 & 47 & 60 \\
\hline Candida-specific Antibodies & Immunodiffusion positive & 8 & 42 \\
\cline { 2 - 5 } & Immunodiffusion negative & 6 & 46 \\
\cline { 2 - 5 } & Total & 14 & 48 \\
\hline
\end{tabular}

significant association between culture-positivity and presence of any risk factor $(\mathrm{p}=0.5)$. There was no difference, as well, in the fungal recovery rates between male and female patients. Similarly, none of the radiological patterns showed significantly higher association with fungal recovery $(\mathrm{p}=0.7)$. Interestingly, even normal chest radiography was associated with fungal growth in 5 of the 10 patients. The growth of Cryptococcus neoformans was associated with reticulo-nodular lesion on chest radiography (Table 4).

\section{Discussion}

The literature is replete with reports of fungal lung infections occurring in the background of overtly immunocompromising conditions like transplantation, haematological malignancy, neutropenia and HIV/AIDS. Though several authors have examined the incidence of infection with specific fungal agents in the context of chronic lung pathology, studies on pulmonary mycoses, as a group, have been relatively limited. In this study we report the profile of fungal colonization of the respiratory tract in patients presenting with different chronic respiratory conditions in a tertiary care teaching hospital located in the Himalayan region of India. We observe that $46.7 \%$ of the patients recruited in our study were culture-positive for fungal agents and 35\% demonstrated presence of anti-fungal precipitin antibodies. Fungal colonization was irrespective of the underlying lung pathology and could not be predicted by the presence of any specific co-morbid condition, risk factor or radiological presentation. Moreover, a normal chest X-ray could not be used to exclude the possibility of accompanying pulmonary fungal infection. Interestingly, the identity of the fungi recovered was different between the major diagnostic categories with significant predilection being observed for colonisation with Aspergillus sp. in patients suffering from Bronchogenic carcinoma and for Candida sp. among patients with Tubercular sequelae.

Though it is difficult to make a confirmed distinction between fungal colonisation of the lung and active fungal infection [1], we considered our patients to be colonised since none of our patients satisfied the criteria for "proven", "probable" or "possible" Invasive Fungal Disease, as proposed in the revised definitions of invasive fungal disease from the European Organization for Research and Treatment of Cancer/Invasive Fungal Infections Cooperative Group and the National Institute of Allergy and Infectious Diseases Mycoses Study Group (EORTC/MSG) Consensus Group [8]. None of these patients showed any radiological abnormality which could not be explained by their underlying lung pathology. Further follow-up study on patient progress is

Table 3 Recovery of fungal agents with respect to different risk factors

\begin{tabular}{cccll}
\hline Co-morbid conditions/risk factors & No. of patients & Culture +ve patients (\%) & Isolates & No. of Isolates (\%) \\
\hline Diabetes mellitus & 7 & $5(71.4)$ & C. albicans & $5(71.4)$ \\
\hline Steroid use & 3 & $2(66.7)$ & C. albicans & $1(33.3)$ \\
\cline { 2 - 5 } & & & A. flavus & $1(33.3)$ \\
\cline { 2 - 5 } & 3 & $2(66.7)$ & A. flavus & $1(33.3)$ \\
\hline Chronic carrier HBV & 1 & $1(100)$ & C. albicans & $1(100)$ \\
\hline Post renal transplant & 1 & $1(100)$ & C. albicans & $1(100)$ \\
\hline Pancytopenia & 3 & $1(33.3)$ & C. albicans & $1(100)$ \\
\hline Alcoholism & 6 & $3(50)$ & C. albicans & $2(33.3)$ \\
\hline Anaemia (Hb $<9 \%)$ & 1 & A. niger & $1(16.7)$ \\
\hline CRF & 1 & C. albicans & $1(100)$ \\
\hline Cirrhosis & $1(100)$ & C. albicans & $1(100)$ \\
\hline
\end{tabular}


Table 4 Association of different fungal isolates with radiological findings

\begin{tabular}{|c|c|c|c|c|}
\hline Radiological finding & No. of patients & Culture +ve patients (\%) & Isolates & No. of Isolates (\%) \\
\hline \multirow[t]{3}{*}{ Consolidation } & 18 & $9(50)$ & C. albicans & $5(27.8)$ \\
\hline & & & A. flavus & $2(11.1)$ \\
\hline & & & A. fumigatus & $2(11.1)$ \\
\hline \multirow[t]{3}{*}{ Collapse } & 9 & $4(44.4)$ & A. flavus & $2(22.2)$ \\
\hline & & & A. fumigatus & $1(11.1)$ \\
\hline & & & C. tropicalis & $1(11.1)$ \\
\hline \multirow[t]{2}{*}{ Nodular lesion } & 8 & $2(25)$ & C. albicans & $1(12.5)$ \\
\hline & & & A. niger & $1(12.5)$ \\
\hline \multirow[t]{3}{*}{ Consolidation + Pleural effusion } & 5 & $3(60)$ & C. tropicalis & $1(20)$ \\
\hline & & & A. flavus & $1(20)$ \\
\hline & & & A. fumigatus & $1(20)$ \\
\hline \multirow[t]{3}{*}{ Normal } & 10 & $5(50)$ & C. albicans & $3(30)$ \\
\hline & & & A. flavus & $1(10)$ \\
\hline & & & A. niger & $1(10)$ \\
\hline Pleural effusion & 2 & $1(50)$ & A. niger & $1(50)$ \\
\hline Fibrotic change + Pleural thickness & 3 & $1(33.3)$ & C. albicans & $1(33.3)$ \\
\hline Cavity & 4 & $2(50)$ & C. albicans & $2(50)$ \\
\hline Reticulonodular lesion & 1 & $1(100)$ & C. neoformans & $1(100)$ \\
\hline
\end{tabular}

required to determine whether such colonization is a prelude to the development of co-morbidity due to invasive or disseminated fungal disease and if so, to work out the time kinetics and nature of such progression. However, none of our patients developed invasive fungal infection till the completion of the study.

Though Candida sp. and Aspergillus sp. constitute the bulk of fungi reported in pulmonary mycoses, their relative proportion and species distribution have shown considerable geographical variation. In the present study, Candida albicans was the most frequent isolate, being recovered from $42.9 \%$ of patients, followed by Aspergillus flavus (21.4\%), Aspergillus fumigatus (14.3\%), Aspergillus niger (10.7\%), Candida tropicalis (7.1\%) and Cryptococcus neoformans (3.6\%). In a similar study on Candida infection in chronic pulmonary conditions, Phukan et al. reported an isolation rate of $50 \%$ from sputum specimens, with $C$. albicans and C. tropicalis predominating [9]. In studies on pulmonary Aspergillosis occurring in chronic lung diseases, Kurhade et al [10] and Shahid et al [11] have reported isolation of the fungus from $16.3 \%$ and $14.7 \%$ of cases of chronic respiratory diseases, using sputum and BAL samples respectively. The species distribution of Aspergillus showed some difference between these studies and the present study. Aspergillus fumigatus accounted for $80 \%$ of the isolates in the former study and Shahid et al [11] reported Aspergillus fumigatus as the predominant species being isolated from $69.2 \%$ cases. Our findings are consistent with reports from other centres in India, in which isolation rate of $A$. flavus largely exceeded that of $A$. fumigatus, underscoring the importance of geographical differences in fungal recovery [12].

The performance of serological tests in the diagnosis of pulmonary mycoses is dependent on a number of factors including the choices of antigen and assay and the immune status of patients. Discordant result between culture and immunodiffusion assays was observed by us in 18 of the 60 (30\%) patients. Among them, immunocompromising conditions were observed in 8 of the 12 patients who were culture-positive but immunodiffusion-negative. In view of the ubiquity of common fungi, serological evidence has been reported to assist in confirming fungal infection and add to the clinical significance of fungal recovery [13]. However, interpretation of serological assays becomes difficult in the presence of immunocompromised clinical status $[10,14]$ and invasive fungal infections [15]. In our study, 5 of the 6 patients who were immunodiffusion-positive but culture-negative had bronchogenic carcinoma. The sixth patient had pleural effusion of unresolved aetiology, in whom the possibility of bronchogenic carcinoma could not be excluded. Fungal recovery may be difficult in bronchogenic carcicoma because of non-communication of the lesion with main bronchus [11] and might have been improved in our study on including multiple specimens. Similar observations have been reported by Malik et al who found serology to be more sensitive than culture for the detection of Aspergillosis in patients with bronchogenic carcinoma [16]. We observed 
seropositivity for Aspergillus antigen in 15\% of recruited patients, which is in broad agreement with figures reported in similar studies $[10,11,13,17,18]$.

Owing to the presence of pre-existing respiratory pathology in the recruited patients, it is difficult to establish a causal relationship between the culture-positivity and radiological abnormality. However, 50 of our patients had radiological abnormalities in their chest $\mathrm{X}$-ray, of whom 23 patients were culture-positive for fungal agents. On the other hand, 5 of our culture-positive patients had normal Chest X-ray. Bronchoscopic examnation of all of these 5 patients revealed the presence of central tumors, which could account for the normal appearance on chest X-ray.

In the present study, we report for the first time, serological evidence of Candidiasis in patients with chronic respiratory diseases. Immunodiffusion test with cytoplasmic antigen of Candida albicans was positive in 12 of the 60 patients $(20 \%)$. Of them, 8 patients were culturepositive for Candida and the remaining 4 patients were culture-negative. Sero-reactivity to the $47 \mathrm{kD}$ cytoplasmic antigen has been shown in previous studies to discriminate between patients with deep-seated invasive Candidiasis and superficial Candidiasis and has been proved to be a valuable adjunct in the diagnosis of invasive candidiasis $[19,20]$. In view of these findings, we used the cytoplasmic antigen of Candida albicans in an immunodiffusion format and detected precipitin antibodies in $57.1 \%$ of our patients.

The point prevalence of fungal colonization was found to be almost $50 \%$ in our patients. Since no association was observed with clinical diagnosis, co-existing risk factors and radiological findings, it is imperative that the possibility of accompanying fungal colonization should be explored meticulously in such patients. An interesting predilection was observed for Aspergillus $s p$. and Candida $s p$. to preferentially infect patients with Bronchoenic carcinoma and Tubercular sequelae respectively. Till further evidence is generated to establish the prognostic implications of fungal colonization with certainty, these patients should be closely monitored, in order to enable earlier detection of invasive or disseminated fungal infection.

\footnotetext{
Acknowledgements

The authors gratefully acknowledge the kind supply of Candida cytoplasmic antigen from Prof Arunaloke Chakrabarti, Deptartment of Medical Microbiology, Post Graduate Institute of Medical Education \& Research, Chandigarh, India.
}

\section{Author details}

${ }^{1}$ Deptartment of Microbiology, Himalayan Institute of Medical Sciences, Swami Ram Nagar, Jolly Grant, Dehradun, Uttarakhand, 248140, India. ${ }^{2}$ Department of Pulmonary Medicine, Himalayan Institute of Medical Sciences, Swami Ram Nagar, Jolly Grant, Dehradun, Uttarakhand, 248140, India.

\section{Authors' contributions}

DB designed the study, supervised the laboratory experiments, analysed the data and drafted the manuscript. SA was involved in performing the laboratory experiments and in data collection and analysis. GS and JR performed the clinical examination and recruitment of the patients, data collection and analysis. All authors have critically reviewed the manuscript, approved of its contents and consented to its communication for publication.

\section{Competing interests}

The authors declare that they have no competing interests.

Received: 30 June 2010 Accepted: 20 September 2010 Published: 20 September 2010

\section{References}

1. Chen KY, Ko SC, Hseueh PR, Luh KT, Yang PC: Pulmonary fungal infection: emphasis on microbiological spectra, patient outcome and prognostic factors. Chest 2001, 120:177-184.

2. Samonis G, Gikas A, Toloudis P, Maraki S, Vrentzos G, Tselentis Y, Tsaparas N, Bodey G: Prospective study of the impact of broad spectrum antibiotics on the yeast flora of the human gut. Eur J Clin Microbiol Infect Dis 1994, 13:665-667.

3. Anaissie E, Solomkin JS: Fungal infection. In Surgical Infections: Diagnosis and treatment. Edited by: Meakins JL. New York: Scientific American; 1994:411-425.

4. Davies SF: Fungal pneumonia. Med Clin North Am 1994, 78:1049-1065.

5. Jewkes J, Kay PH: Pulmonary aspergillosis: Analysis of prognosis in relation to hemoptysis and survey of treatment. Thorax 1983, 38:572-578.

6. el-Ebiary M, Torres A, Fàbregas N, de la Bellacasa JP, González J, Ramirez J, del Baño D, Hernández C, Jiménez de Anta MT: Significance of the isolation of Candida species from respiratory samples in critically ill, non-neutropenic patients. An immediate postmortem histologic study. Am J Respir Crit Care Med 1997, 156:583-590.

7. Jourdain B, Joly-Guillou ML, Dombert MC, Calvat S, Trouillet JL, Gibert C, Chastre J: Usefulness of quantitative cultures of BAL fluid for diagnosing nosocomial pneumonia in ventilated patients. Chest 1997, 111:411-418.

8. De Pauw B, Walsh TJ, Donnelly JP, Stevens DA, Edwards JE, Calandra T, Pappas PG, Maertens J, Lortholary O, Kauffman CA, Denning DW, Patterson TF, Maschmeyer G, Bille J, Dismukes WE, Herbrecht R, Hope WW, Kibbler CC, Kullberg BJ, Marr KA, Muñoz P, Odds FC, Perfect JR, Restrepo A, Ruhnke M, Segal BH, Sobel JD, Sorrell TC, Viscoli C, Wingard JR, Zaoutis T, Bennett JE, European Organization for Research and Treatment of Cancer/ Invasive Fungal Infections Cooperative Group; National Institute of Allergy and Infectious Diseases Mycoses Study Group (EORTC/MSG) Consensus Group: Revised definitions of invasive fungal disease from the European Organization for Research and Treatment of Cancer/Invasive Fungal Infections Cooperative Group and the National Institute of Allergy and Infectious Diseases Mycoses Study Group (EORTC/MSG) Consensus Group. Clin Infect Dis 2008, 46:1813-1821.

9. Phukan AC, Sarmabardoloi JN, Mahanta J: Bronchopulmonary candidiasis in a tertiary referral hospital of Assam India. Ind J Med Sci 2000, 54:491-494.

10. Kurhade AM, Deshmukh JM, Fule RP, Chande C, Akulwar S: Mycological and serological study of pulmonary aspergillosis in Central India. Ind J Med Microbiol 2002, 20:141-144.

11. Shahid M, Malik A, Bhargava R: Prevalence of aspergillosis in chronic lung diseases. Ind J Med Microbiol 2001, 19:201-205.

12. Saravanan K, Panda NK, Chakrabarti A, Das A, Bapuraj RJ: Allergic Fungal Rhinosinusitis An Attempt to Resolve the Diagnostic Dilemma. Arch Otolaryngol Head Neck Surg 2006, 132:173-178.

13. Vijaychari P, Lakshmi A, Gururaj AK: Serodiagnosis of pulmonary aspergillosis. Ind J Med Microbiol 1997, 15:23-24.

14. Saugier-Veber $P$, Devergie A: Epidemiology and diagnosis of invasive pulmonary aspergillosis in bone marrow transplant patients, results of a 5 year retrospective study. Bone Marrow Transplant 1993, 12:121-124.

15. Manso $E$, Montillo $G$ : Value of antigen and antibody detection in the serological diagnosis of invasive aspergillosis in patients with hematological malignancies. Eur J Clin Microbiol Infect Dis 1994, 13:756-760. 
16. Malik A, Shahid M, Bhargava R: Prevalence of aspergillosis in bronchogenic carcinoma. Ind J Pathol Microbiol 2003, 46:507-510.

17. Rangaswamy V, Vasantha KR, Seshagiri K: Serological diagnosis of pulmonary aspergillosis. Ind J Pathol Microbiol 1977, 20:101-104.

18. Shivananda PG: Pulmonary aspergillosis. ICMR Bulletin 1992, 22:107-108.

19. Porsius JC, Vanvliet HJ, Van Zeifi JH, Gossens WH, Michel HF: Detection of an antibody response in immunocompetent patients with systemic candidiasis or Candida albicans colonization. Eur J Clin Microbiol Infect Dis 1990, 9:352-355.

20. Chakrabarti A, Roy P, Kumar D, Sharma BK, Chugh KS, Panigrahi D: Evaluation of three serological test for detection of anticandidial antibodies in diagnosis of invasive candidiasis. Mycopathologia 1994, 12:3-7.

doi:10.1186/1476-0711-9-28

Cite this article as: Biswas et al.: Fungal colonization in patients with chronic respiratory diseases from Himalayan region of India. Annals of Clinical Microbiology and Antimicrobials 2010 9:28.

\section{Submit your next manuscript to BioMed Central and take full advantage of:}

- Convenient online submission

- Thorough peer review

- No space constraints or color figure charges

- Immediate publication on acceptance

- Inclusion in PubMed, CAS, Scopus and Google Scholar

- Research which is freely available for redistribution

Submit your manuscript at www.biomedcentral.com/submit 